\title{
Mesenchymal tumors of the prostate
}

\author{
Jesse K McKenney \\ Robert J Tomsich Pathology and Laboratory Medicine Institute, Anatomic Pathology, Cleveland Clinic, \\ Cleveland, $\mathrm{OH}, \mathrm{USA}$
}

\begin{abstract}
Mesenchymal tumors of the prostate are rare but often cause considerable diagnostic difficulty when encountered. These may be either benign or malignant and can arise within the prostate gland or in adjacent tissues. This review covers prostatic stromal proliferations (prostatic stromal tumor of uncertain malignant potential and stromal sarcoma), solitary fibrous tumor, myofibroblastic proliferations, smooth muscle neoplasms (leiomyoma and leiomyosarcoma), gastrointestinal stromal tumor, schwannoma, rhabdomyosarcoma, sarcomatoid carcinoma, postradiation sarcoma, and mixed epithelial stromal tumor of the seminal vesicle. Differential diagnostic considerations are emphasized, including descriptions of helpful histological and/or clinical clues and the use of adjuvant diagnostic techniques.

Modern Pathology (2018) 31, S133-S142; doi:10.1038/modpathol.2017.155
\end{abstract}

True mesenchymal neoplasms of the prostate make up $<1 \%$ of the tumors occurring in this anatomical location. Considerable diagnostic difficulties may be encountered with such lesions due to their rarity, which leads to a very limited experience for most pathologists. This is further complicated by the fact that these lesions are sampled by relatively small core biopsy specimens and that the diagnosis is often unexpected. Moreover, the histological differences between distinct spindle cell lesions are generally more subtle than for epithelial neoplasms. Although prostatic stromal proliferations/neoplasms represent the most commonly considered mesenchymal tumor at this site, solitary fibrous tumor, myofibroblastic proliferations, smooth muscle neoplasms, gastrointestinal stromal tumors, schwannomas, rhabdomyosarcomas, postradiation sarcomas, and mixed epithelial stromal tumors of the seminal vesicle may all be encountered in 'prostate biopsies'. Sarcomatoid carcinoma, while not of mesenchymal derivation, is also discussed due to its considerable histological overlap with sarcomas. This review details the diagnostic features of each entity to include detailed differential diagnostic considerations and helpful adjunctive diagnostic tests.

Correspondence: Dr JK McKenney, MD, Robert J. Tomsich Pathology and Laboratory Medicine Institute, Anatomic Pathology, Cleveland Clinic, 9500 Euclid Avenue, L15, Cleveland, OH 44195, USA.

E-mail: mckennj@ccf.org

Received 30 August 2017; revised 19 September 2017; accepted 21 September 2017

\section{Prostatic stromal proliferations}

Benign prostatic hyperplasia (BPH), which is often stromal predominant, is a fairly common finding in transurethral resection specimens of the prostate. As florid BPH must be differentiated from prostate stromal neoplasia, the latter is probably the most frequently considered spindle cell neoplasm of the prostate gland. The current WHO classification includes two diagnostic categories: stromal tumor of uncertain malignant potential (STUMP) and stromal sarcoma. ${ }^{1}$

Prostatic STUMPs may have significant histological overlap with florid stromal hyperplasia. Several histological patterns of STUMP are described, including cellular spindled, eosinophilic pattern (closely mimicking smooth muscle), a pattern with scattered atypical stromal cells, phyllodes-like pattern, and myxoid pattern (Figures 1a-d); these patterns may be intermixed. ${ }^{2-4}$ Another recently described histological pattern has 'round cell' morphology. ${ }^{5}$ The hypercellular stromal pattern consists of bland fusiform stromal cells, often admixed with benign prostatic glands. In general, the cytologically bland spindle cell pattern has the most overlap with stromal hyperplasia. Stromal hyperplasia generally contains small blood vessels, often with some degree of hyalinization, which is distinctive and helps in distinction from a true prostatic stromal neoplasm (Figures 2a and b). On low power evaluation, florid stromal hyperplasia often has a more lobulated or nodular architecture, while a true prostatic stromal neoplasm (STUMP) should have a more diffuse growth pattern. It should be noted that BPH can occasionally have stromal 

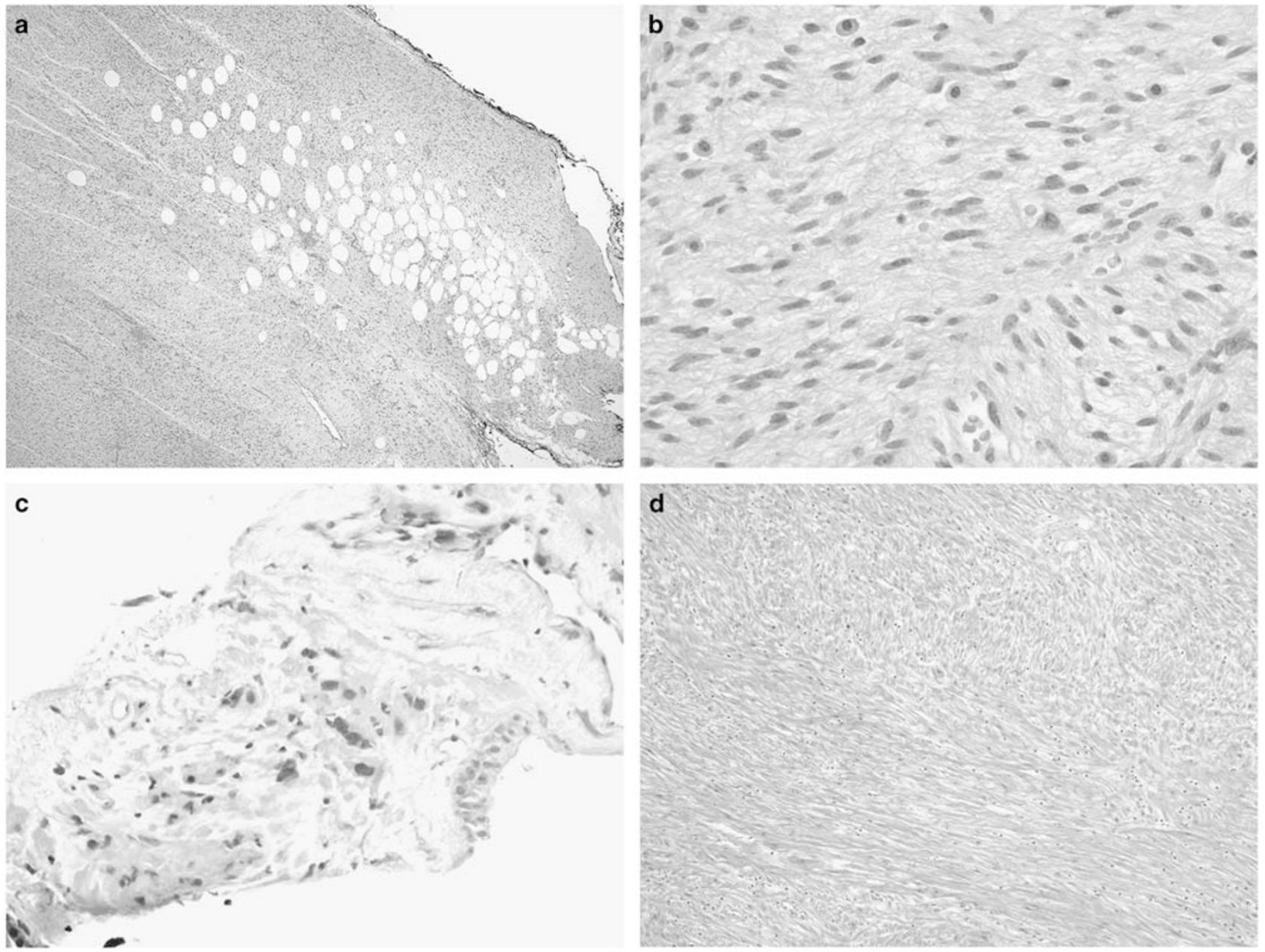

Figure 1 Prostatic stromal neoplasm of uncertain malignant potential (STUMP) with (a) cellular spindled pattern and invasion of extraprostatic soft tissue. (b) The individual neoplastic cells are cytologically bland. Other patterns include (c) tumors with scattered atypical stromal cells between glands and (d) an eosinophilic pattern mimicking smooth muscle.
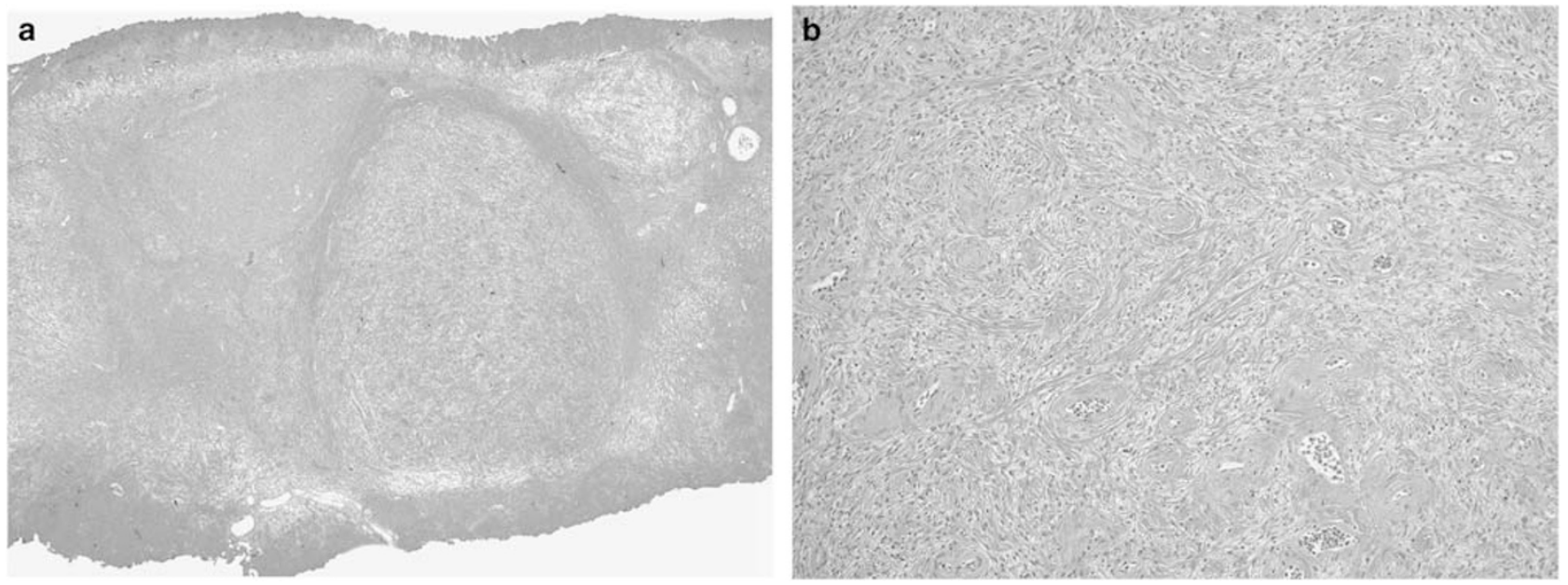

Figure 2 Benign prostatic hyperplasia (BPH) may be distinguished from STUMP by (a) lobular/nodular architecture and (b) a characteristic vascular pattern with small round well-formed blood vessels.

indentation into a glandular space creating a 'phyllodes-like' or 'fibroadenoma-like' architecture (Figure 3a). In such cases, the hyperplastic stroma does not show cellular condensation adjacent to the epithelium, and this 'phyllodes-like' pattern is often very focal in the background context of prototypical BPH. Myxoid patterns of prostatic STUMP may mimic a variety of other myxoid mesenchymal 

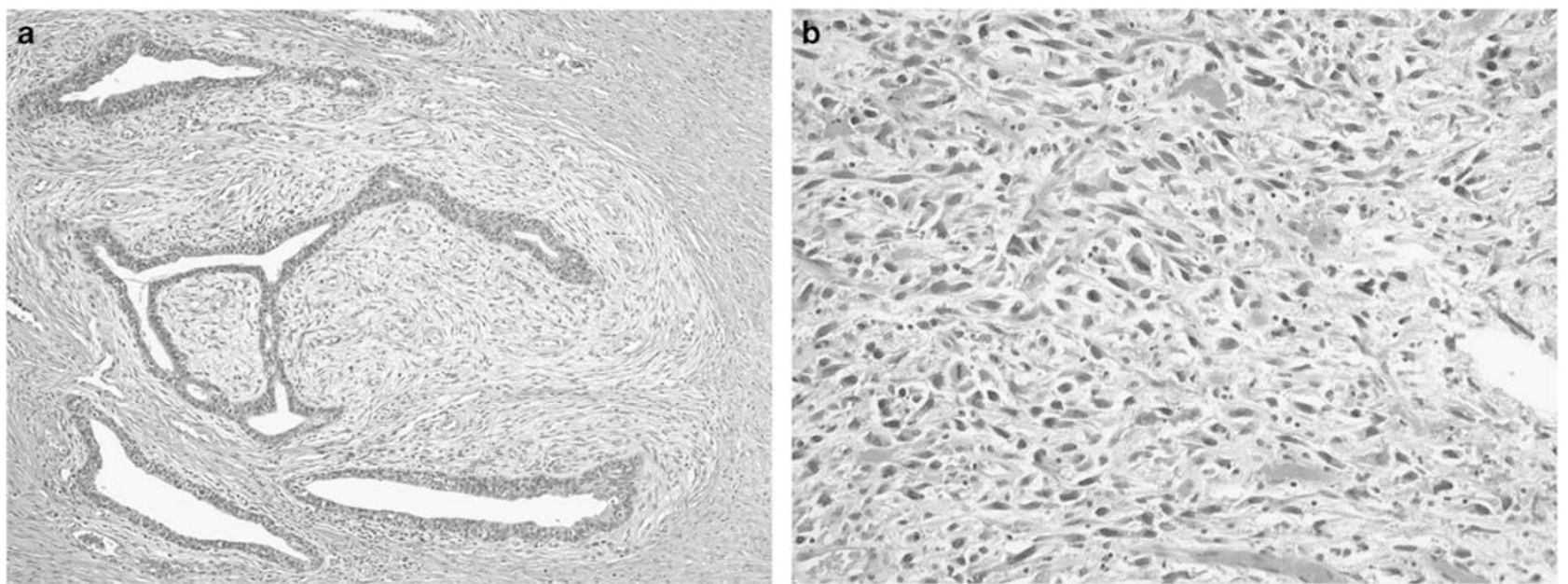

Figure 3 (a) Benign prostatic hyperplasia (BPH) may have foci that mimic a 'phyllodes-like' pattern, but other surrounding features of BPH are typical. (b) Prostatic stromal sarcoma has marked nuclear atypia and mitotic activity is often brisk.

lesions, including inflammatory myofibroblastic tumor and myxoid variant of solitary fibrous tumor. The 'round cell' pattern of STUMP may be more readily mistaken for a gastrointestinal stromal tumor (GIST), or possibly a prostatic adenocarcinoma, but immunostains should easily resolve those considerations (discussed in separate sections below).

Epithelial proliferations are also described in association with prostatic STUMPs and include: glandular crowding, prominent basal cells, papillary infolding, cystic dilatation, basal cell hyperplasia, urothelial metaplasia, squamous metaplasia, and cribriform hyperplasia. ${ }^{6}$ Of these features, glandular crowding and prominent basal cells are most common.

Prostatic stromal sarcomas are much easier to diagnosis as neoplastic because their overtly malignant features are more easily distinguished from florid stromal hyperplasia. Stromal sarcomas often show greater cellularity, greater cytological atypia, easily identifiable mitotic activity, and tumor cell necrosis (Figure 3b). When phyllodes-like patterns are present, there is often marked stomal condensation around the glands, a pattern similar to a malignant phyllodes tumor of the breast or adenosarcoma of the uterus.

Immunophenotypically, prostatic stromal tumors commonly show expression of CD34 and progesterone receptor. Although some actin and desmin reactivity may be seen in STUMPs, this is commonly lost in stromal sarcomas. Additionally, while not commonly used for diagnosis, common chromosomal aberrations are reported and include loss of chromosome 10, 13, and $14 .^{7}$

Prostatic STUMPs are typically indolent and are generally cured with complete resection. They may be treated more conservatively in carefully considered patients; however, there are rare cases associated with sarcomatous dedifferentiation, warranting very careful clinical follow-up if resection is not attempted. Sarcomas have fully metastatic potential and generally require surgical resection and consideration for adjuvant therapy.

\section{Solitary fibrous tumor}

Solitary fibrous tumor represents a distinct fibroblastic-type mesenchymal neoplasm that may have significant histological overlap with prostatic stromal neoplasia and florid stromal hyperplasia. In the older literature, many of these tumors were diagnosed and reported as 'hemangiopericytoma', but they are now designated as solitary fibrous tumor. ${ }^{8}$ Given the common expression of CD34 by solitary fibrous tumors at any anatomical site, there is significant overlap with prostatic stromal proliferations. In fact, there was original skepticism about true solitary fibrous tumors occurring in the prostate. However, identification of a novel recurrent in NAB2-STAT6 gene fusion has confirmed that solitary fibrous tumors do occur in the prostate (and it also provides a very useful diagnostic signature). ${ }^{9,10}$ These tumors appear identical to their description in other anatomical sites, with bland fibroblast-like cells arranged in a very disorganized non-repetitive pattern (ie, 'patternless' pattern), scattered angulated hemangiopericytic-like vessels, variable stromal collagenization, and occasional cellular and myxoid forms (Figures 4a and b). Occasionally, a cord-like growth pattern may be seen and could mimic a smooth muscle neoplasm or even a plasmacytoid carcinoma from the urothelium. Rare solitary fibrous tumors may contain lipid-rich areas. In superficial biopsies obtained by a transurethral method, solitary fibrous tumor can be difficult to recognize and may closely mimic benign subepithelial connective tissue or BPH. A novel monoclonal antibody for STAT6 is now available, which shows strong and diffuse nuclear reactivity in solitary fibrous tumors and is generally negative in most morphological mimics. ${ }^{11-13}$ Although some immunophenotypic 

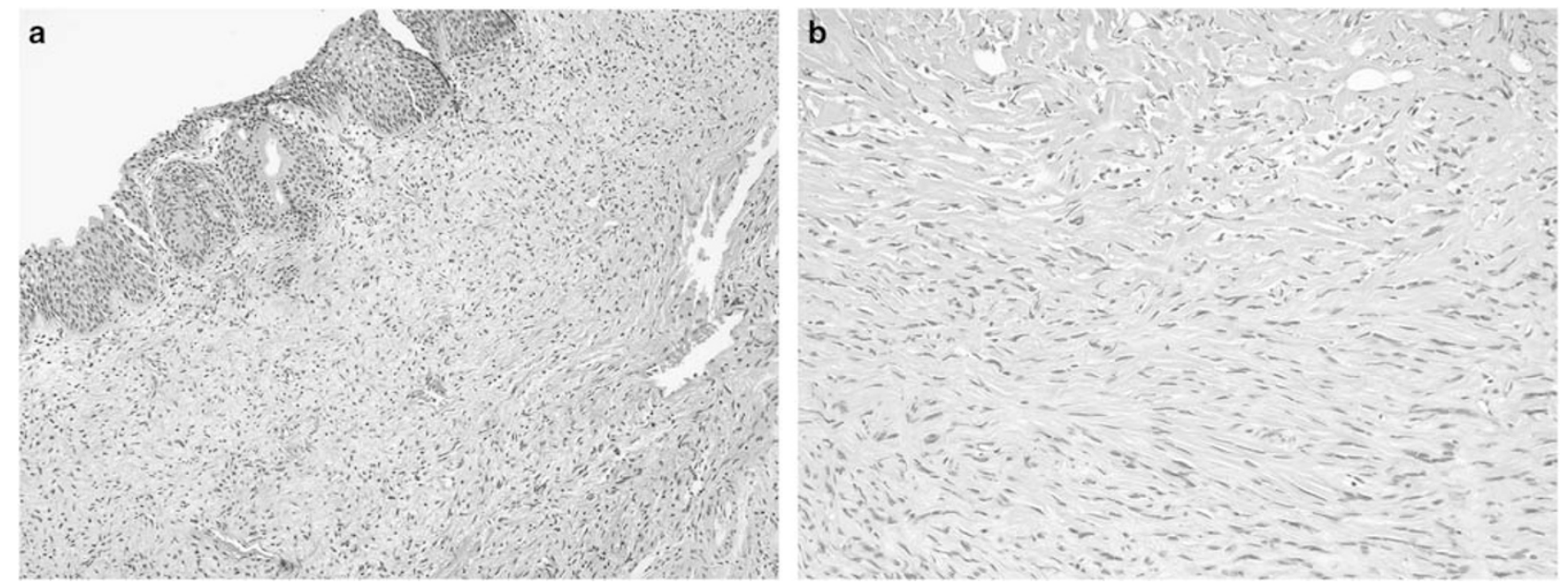

Figure 4 Solitary fibrous tumor (a) may extend to the urethral surface where it could mimic lamina propria or BPH. (b) Some foci may show more characteristic intercellular collagen.

overlap is reported between solitary fibrous tumor and prostatic stromal neoplasms using the polyclonal STAT6 antibody, ${ }^{14}$ it is our experience that the monoclonal antibody is more specific. However, this warrants further study, and fluorescence in situ hybridization (FISH) or PCR confirmation of the NAB2-STAT6 fusion is available for challenging cases. It should also be remembered that STAT6 may be amplified in a subset of dedifferentiated liposarcomas; therefore, those tumors may also show nuclear reactivity with the STAT6 antibody. ${ }^{15,16}$ Finally, it is important for genitourinary pathologists to be aware that solitary fibrous tumor expresses PAX-8 in $40-45 \%$ of cases. ${ }^{17}$

Solitary fibrous tumors, when completely excised, most commonly follow a benign clinical course, but more aggressive behavior can occur. ${ }^{18}$ There are several prognostic models provided for risk assessment in solitary fibrous tumors and they generally include features such as tumor size, anatomical location, mitotic activity, nuclear pleomorphism, necrosis, and patient age. ${ }^{19-21}$

\section{Smooth muscle neoplasms}

Smooth muscle neoplasms in the prostate are similar to those that occur elsewhere, such as somatic soft tissue and the uterus. Leiomyomas are rare and their distinction from prostatic stromal hyperplasia is arbitrary in some cases. They are comprised of a well-circumscribed proliferation of cytologically bland spindle cells with prominent eosinophilic cytoplasm and a fascicular architecture. Leiomyosarcomas may be infiltrative or have a pushing border but show greater nuclear variability, nuclear hyperchromasia (Figure 5), mitotic activity, and (in some cases) necrosis. As in other sites, leiomyosarcomas are aggressive with potential for distant metastasis.

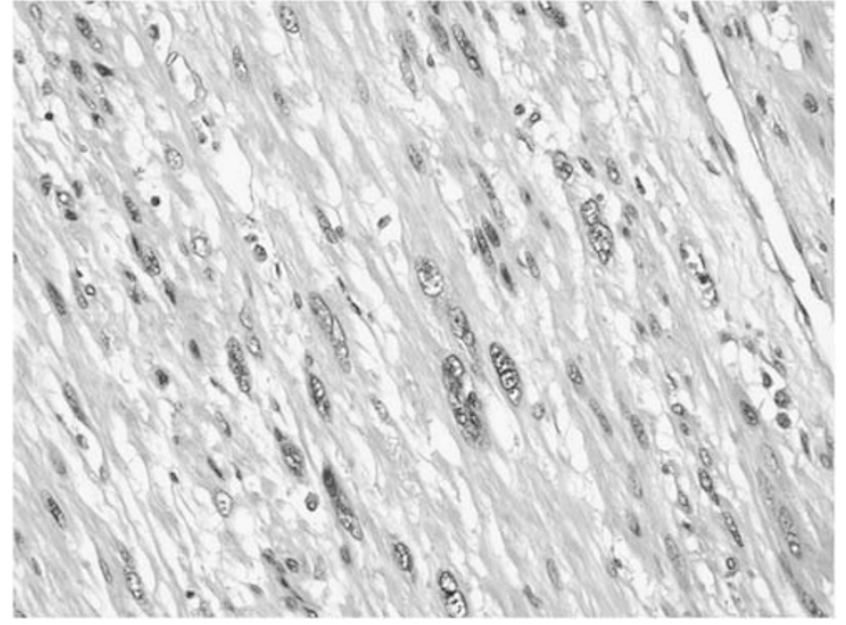

Figure 5 Leiomyosarcoma typically has marked nuclear atypia.

There are some reported cases of 'atypical' leiomyomas in the urinary tract, analogous to those in the uterus. ${ }^{22,23}$ Although we acknowledge that rare smooth muscle neoplasms of the urinary tract, including the prostate gland, may have morphological features similar to 'atypical' (also known as 'symplastic' or 'bizarre') leiomyoma, that diagnosis should be rendered with extreme caution. These tumors may have significant overlap with the atypical cell pattern of prostatic STUMP. For a smooth muscle tumor with cytological atypia, the presence of mitotic activity would favor a leiomyosarcoma.

\section{Myofibroblastic proliferations}

Myofibroblastic proliferations may occur in the prostate but are much less common than those seen in the urinary bladder. These have been described under a variety of different names, such as postoperative spindle cell nodule, inflammatory 
myofibroblastic tumor, and pseudosarcomatous myofibroblastic proliferation. ${ }^{24-27}$ Some occur de novo, while others follow a prior injury (including biopsy). Cases described as inflammatory myofibroblastic tumor have heterogeneous findings, but up to $75 \%$ have an $A L K$ re-arrangement by FISH and ALK protein expression by immunohistochemistry. ${ }^{24,28}$ Although nomenclature is still debated for myofibroblastic proliferations in the urinary tract, cases with typical histological features have all behaved in a benign manner regardless of prior instrumentation or presence/absence of $A L K$ re-arrangement. Immunophenotypically, these myofibroblastic proliferations often express low-molecular-weight cytokeratin, which may cause confusion with sarcomatoid carcinoma; however, they do not express high-molecular-weight cytokeratins. They also commonly express smooth muscle actin and desmin.

The most important differential diagnostic distinction is with a malignant neoplasm, such as leiomyosarcoma (or other sarcoma) and sarcomatoid carcinoma with myxoid stroma. Myofibroblasts may have significant nuclear variability in size and shape, and prominent nucleoli (sometimes multiple) are not uncommon, but the chromatin is evenly dispersed (Figures 6a and b). The presence of irregular, hyperchromatic chromatin is the key feature that suggests a malignant neoplasm. ${ }^{29}$ In patients aged $<40$ years of age, we would recommend careful exclusion of embryonal rhabdomyosarcoma before a myofibroblastic lesion is diagnosed (see below).

\section{Gastrointestinal stromal tumor}

Very rarely, GISTs may be sampled on transrectal prostate biopsies. ${ }^{30}$ GISTs discovered in prostate needle biopsies often represent rectal based tumors that compress and displace the prostate gland. These
GISTs, similar to other sites of origin, may have a variety of histological patterns, including spindled and epithelioid morphology (Figure 7). ${ }^{31}$ The spindle cell morphology may have significant overlap with that of prostatic stromal proliferations, which are more common. Immunophenotypically, GISTs characteristically express CD34, CD117, and DOG-1. Although not exhaustively studied, DOG1 staining has been reported in prostatic stromal sarcoma. ${ }^{32}$ As with many tumors presenting in unusual locations, the key to diagnosis is routinely considering the diagnostic possibility of GIST in this location when faced with a spindle cell lesion. Although not specifically evaluated in the prostatic region, we would utilize the typical GIST prognostic stratification recommend by the WHO. ${ }^{31}$

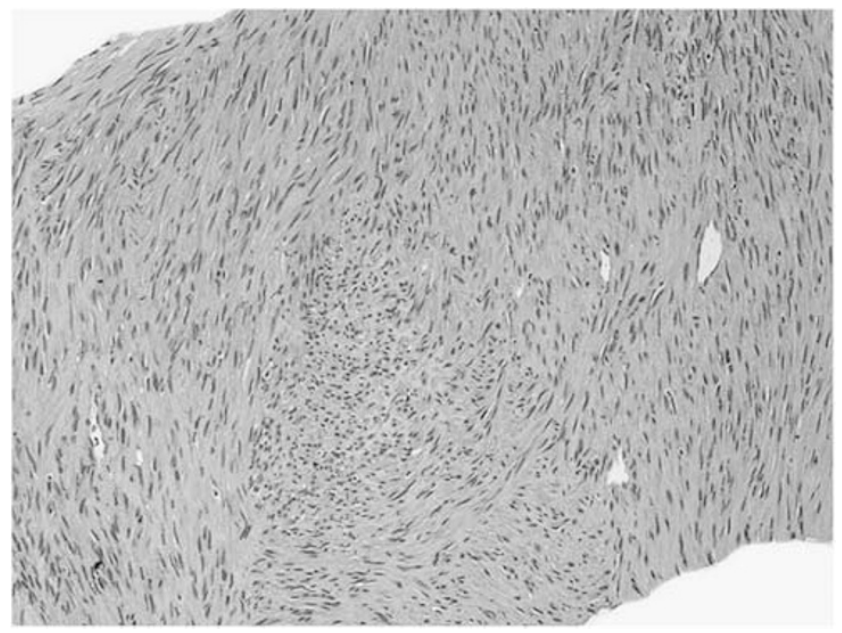

Figure 7 Gastrointestinal stromal tumor in a prostate needle core biopsy. One must remember to consider this diagnostic possibility in prostate biopsies.
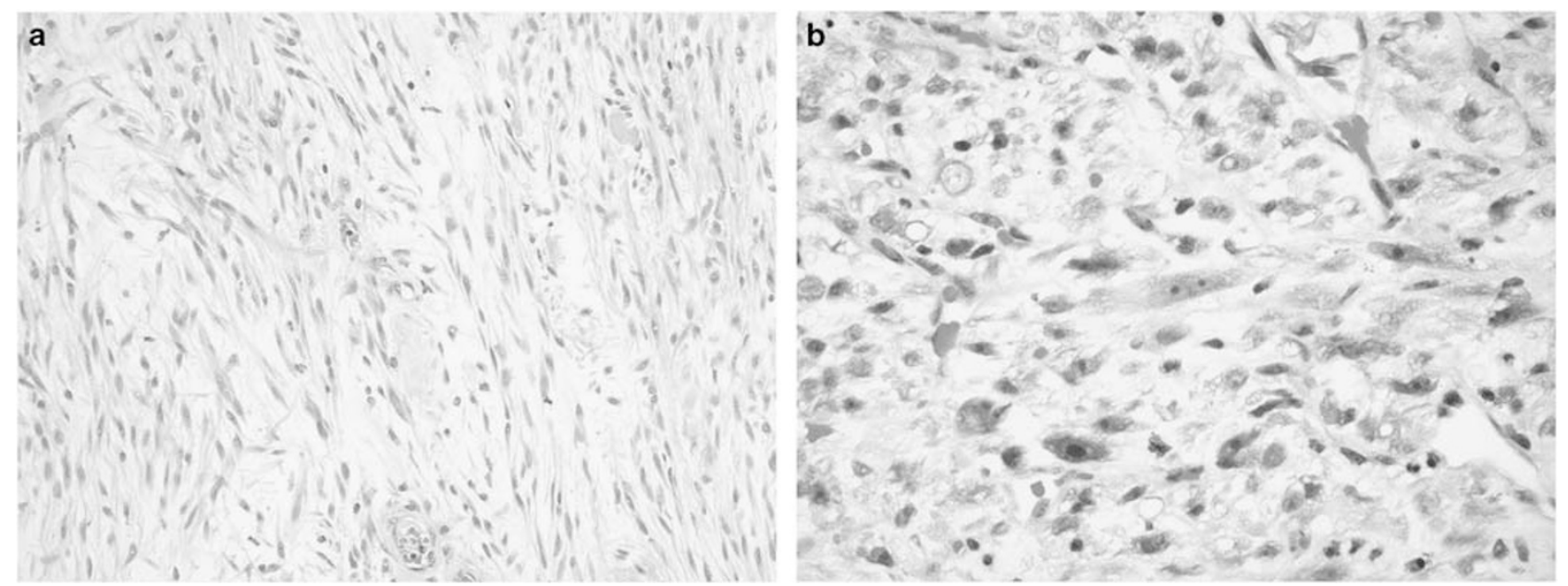

Figure 6 Myofibroblastic proliferation ('inflammatory myofibroblastic tumor') of the prostate. (a) The cells have typical myofibroblastic features with elongated or 'stretched-out' spindle cells, often with a loose myxoid background. (b) The nuclei may have significant variation in size, and nucleoli may be quite prominent or multiple; however, nuclear hyperchromasia is not seen. 


\section{Schwannoma}

The diagnosis of schwannoma is typically straightforward. However, when sampled unexpectedly in a needle core biopsy of the 'prostate' or 'seminal vesicle', the diagnostic possibility of schwannoma may be not be considered. On imaging, a pelvicbased schwannoma may compress the prostate and seminal vesicles, creating confusion as to the site of origin. ${ }^{33}$ There may be significant histological overlap with a prostatic stromal proliferation or solitary fibrous tumor, but helpful histological clues for schwannoma include large caliber blood vessels with mural hyalinization, nuclear palisading, a 'wavy' character of the spindle cells, and variation from hypercellular to hypocellular zones (Figure 8a). Once the diagnosis is considered, immunohistochemical demonstration of diffuse S-100 protein (or SOX-10) expression can confirm the diagnosis. Some schwannomas may appear macroscopically cystic, which causes further overlap with prostatic or seminal vesicle tumors. ${ }^{34,35}$ Histologically, these cystic schwannomas can contain 'pseudoglandular' spaces that are lined by elongated cells resembling pseudostratified columnar epithelium (Figure 8b). ${ }^{36}$ These cyst-lining cells also express S-100 protein and are negative for keratin. One other immunohistochemical pitfall is the expression of PSMA by schwannomas, which may also be detected by PSMA imaging methodologies. ${ }^{37-39}$

\section{Rhabdomyosarcoma}

Rhabdomyosarcoma involving the prostate gland is typically of the embryonal subtype and occurs predominantly in the pediatric population; only rare cases are described in adults. Alveolar, spindle cell/ sclerosing, and pleomorphic variants of rhabdomyosarcoma are extremely rare in this location. These
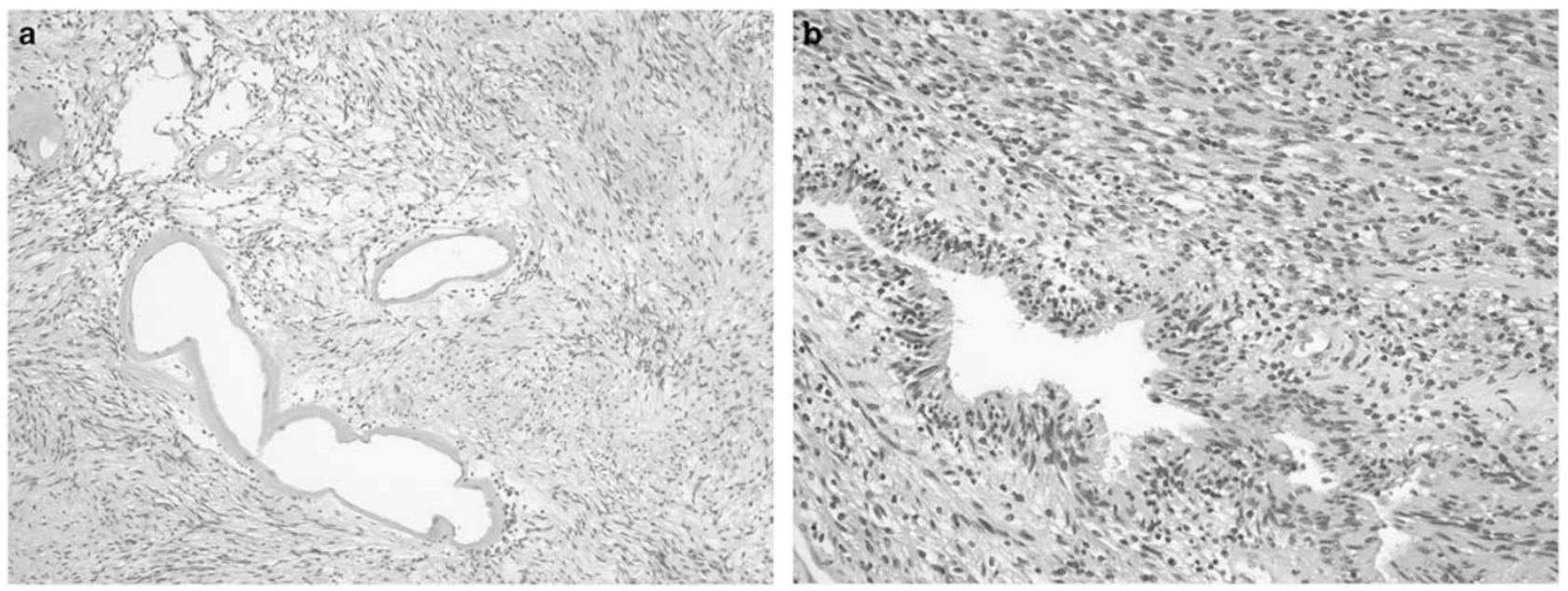

Figure 8 Schwannoma presenting as a 'prostate' mass. (a) The large hyalinized blood vessels are a helpful clue to consider schwannoma. (b) Rare cases show 'pseudoglands' that are not epithelial but may cause consideration of a mixed glandular and stromal lesion.
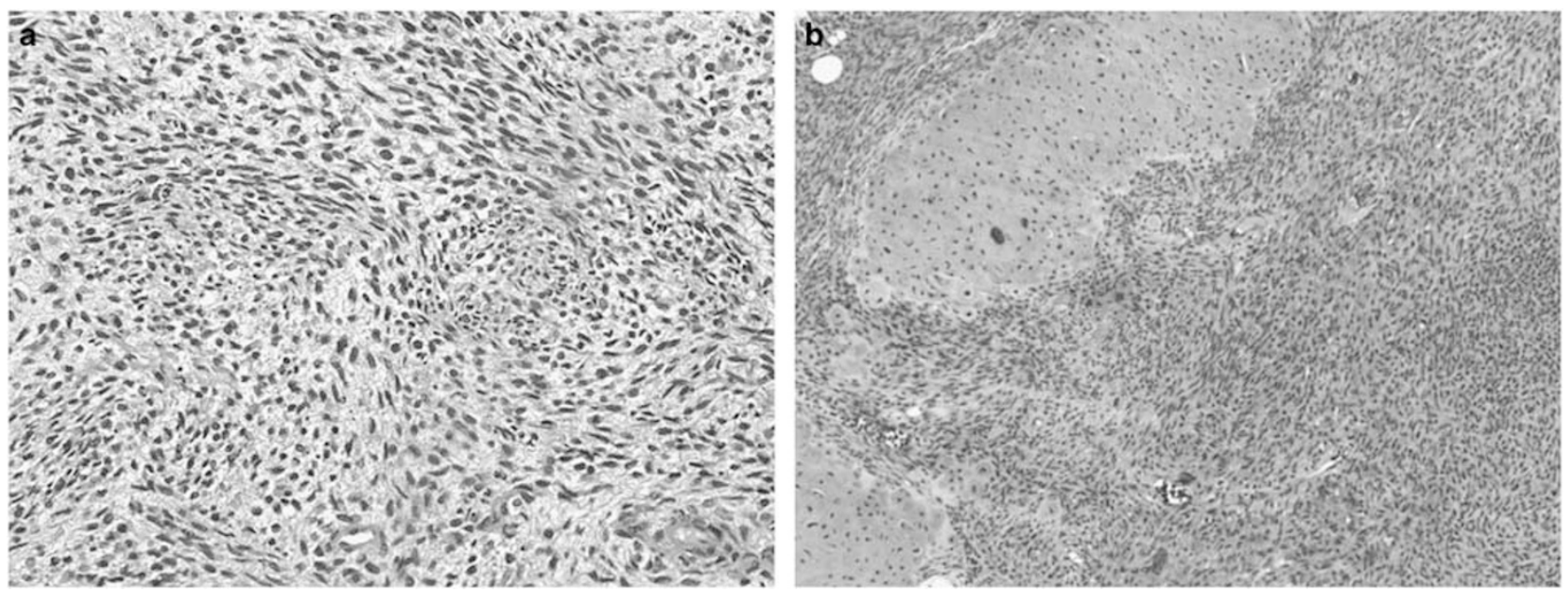

Figure 9 Embryonal rhabdomyosarcoma of the urinary tract (a) may mimic a variety of spindle cell lesions and must be carefully considered in young patients. (b) Cartilaginous differentiation may also be seen. 
tumors often have increased cellularity underneath the prostatic urethra, which may be urothelial or squamous-lined in children; however, this classic cambium layer is not always present because significant morphological heterogeneity is typical. The neoplastic cells are often spindled, but fusiform or more rounded cells may be seen and welldeveloped rhabdomyoblasts are frequently identified (Figure 9a). Some foci may be quite hypocellular and cytological atypia may not be pronounced (Figure 9b); therefore, it is imperative to consider the diagnosis of a rhabdomyosarcoma in young patients when a spindle cell proliferation is encountered on biopsy. Another unusual feature of embryonal rhabdomyosarcoma is the occasional presence of cartilaginous differentiation (Figure 9b), which we have seen in the prostate where it mimics a sarcomatoid carcinoma. ${ }^{40,41}$ Immunohistochemical markers of skeletal muscle differentiation, myogenin and MyoD1, are most helpful for this diagnosis. Correct diagnosis is critical because pediatric rhabdomyosarcoma of the genitourinary tract has excellent cure rates with appropriate standard therapy, which is frequently updated by the Children's Oncology Group. ${ }^{42,43}$

\section{Sarcomatoid carcinoma}

Sarcomatoid carcinoma of the prostate is very rare and may occur de novo or as an aggressive recurrence following a history of typical acinar adenocarcinoma. ${ }^{44-46}$ Histologically, a mixture of frank epithelial and sarcomatous components are seen in most cases (Figure 10a). The epithelial components often have a high-grade acinar morphology, but ductal, small cell, or squamous differentiation may also be seen. The epithelium expresses markers typical of their differentiation (eg, keratin, NKX3.1, PSA). The sarcomatous component is often 'undifferentiated' with bizarre giant cells (Figure 10b) or pleomorphic spindle cells, but heterologous differentiation in the form of osteosarcoma, chondrosarcoma, and rhabdomyosarcoma are described. In rare cases, epithelial differentiation may not be recognizable histologically and is only documented by immunohistochemistry (in the clinical context of a prior prostate cancer). Immunophenotypically, the sarcomatoid component of most cases will show at least focal expression with broad spectrum keratins, but PSA expression is typically absent. ${ }^{44}$ The main differential diagnostic consideration in a de novo setting is a primary sarcoma. In the setting of prior prostate cancer, a postradiation sarcoma should be considered (see below). Rarely, sclerosing adenosis might mimic a sarcomatoid carcinoma. ${ }^{47-49}$

\section{Postradiation sarcoma}

Postradiation sarcomas often occur close to 10 years following radiation treatment and may show a variety of morphological patterns, most commonly osteosarcoma, pleomorphic undifferentiated sarcoma, and angiosarcoma. ${ }^{50,51}$ The pathological distinction between a sarcomatoid carcinoma and a postradiation sarcoma may be impossible in some cases. Late progression to an aggressive prostatic carcinoma variant may occur (eg, sarcomatoid carcinoma or 'pleomorphic giant cell carcinoma'), which can follow radiation in some cases and often has significant histological overlap with sarcoma. ${ }^{52}$ Obviously, the presence of a more typical carcinomatous component (described above) makes the diagnosis straightforward, but in tumors with a pure sarcomatous pattern adjunctive testing may be needed. Unfortunately, the spindle cell component of sarcomatoid carcinoma may lose the expression of epithelial- and prostatic-specific markers. In the
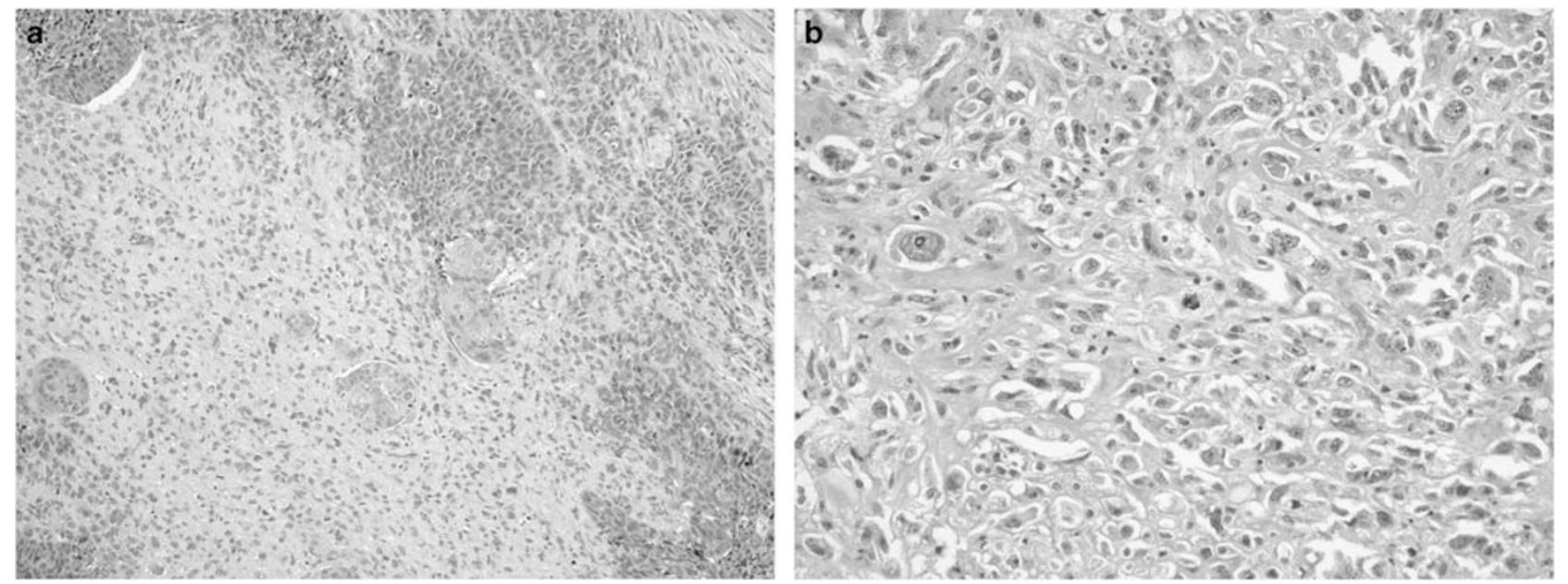

Figure 10 Sarcomatoid carcinoma of the prostate often shows (a) frank carcinomatous and sarcomatous components. The carcinomatous component shows squamous differentiation in this example. (b) Some cases may show markedly pleomorphic tumor giant cells. 

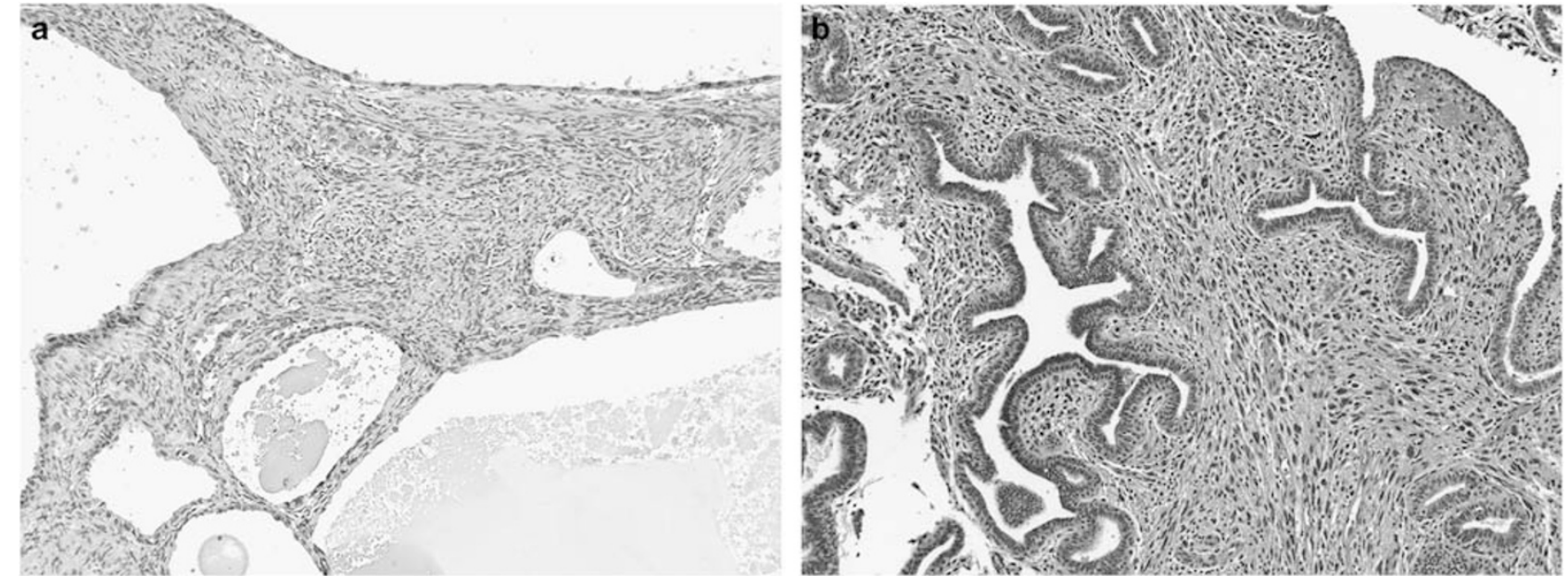

Figure 11 (a) Benign mixed epithelial and stromal tumor of the seminal vesicle shows an admixture of cytologically bland stromal cells and simple epithelial glands, while (b) malignant mixed epithelial and stromal tumor has more marked cytological atypia in the stromal cells.

absence of any histological or immunophenotypic evidence of epithelial differentiation, a postradiation sarcoma may be favored. This distinction makes little clinical difference as both tumors follow a rapidly progressive course despite therapy.

\section{Mixed epithelial-stromal tumor of seminal vesicle}

Mixed epithelial and stromal tumors of the seminal vesicle are rare biphasic neoplasms comprised of benign epithelial glands and more variable stromal components (Figure 11a). They have historically been described under a variety of names, including phyllodes tumor, cystadenoma, cystomyoma, and müllerian adenosarcoma-like tumor. ${ }^{53}$ These may be small incidental lesions seen at radical prostatectomy or may present as large cystic masses. Histologically, benign tumors have a cytologically bland spindle cell stroma separated by cystically dilated, often branching glands. The epithelium is immunoreactive for keratins and negative for PSA, while the stroma commonly expresses smooth muscle actin. Malignant mixed epithelial and stromal tumors are more cellular (often condensing under the epithelium), have more cytological atypia, and have increased mitotic activity (Figure 11b). These malignant tumors may also have areas of sarcomatous stromal overgrowth very similar to adenosarcomas of the gynecological tract. Benign tumors are cured by excision, but the rare malignant variant has potential for an aggressive course with metastatic spread.

In our experience, the most significant differential diagnosis on biopsy is prostatic cystadenocarcinoma. ${ }^{54}$ These may also present as a large cystic masses and may distort the seminal vesicle, causing confusion as to the site of origin. On biopsy, the epithelium is quite heterogenous and may be difficult to recognize as neoplastic. If intracystic papillary excrescences are present (often lined by pseudostratified columnar epithelium), a cystadenocarcinoma of prostatic origin is likely; this may be confirmed with prostatic epithelial markers, such as NKX3.1 or PSA. In contrast, seminal vesicle epithelium lacks this expression and commonly shows nuclear PAX-8 reactivity. ${ }^{55}$ Pseudoglandular schwannomas may also closely mimic a mixed epithelial and stromal tumor of the seminal vesicle (discussed separately above). ${ }^{34,35}$

\section{Other lesions}

A variety of other mesenchymal and spindle cell lesions have been reported to occur very rarely in the prostate. These include: angiosarcoma, ${ }^{56}$ hemangioma, ${ }^{57,58}$ hemangioendothelioma, ${ }^{59}$ pleomorphic undifferentiated sarcoma, ${ }^{60,61}$ synovial sarcoma, ${ }^{62}$ malignant peripheral nerve sheath tumor, ${ }^{63,64}$ neurofibroma, ${ }^{65}$ and granular cell tumor. ${ }^{66}$

\section{Conclusions}

Mesenchymal neoplasms of the prostate may present significant diagnostic difficulty because their morphological features have a significant degree of overlap. Moreover, they are quite rare and often encountered unexpectedly in biopsy or transurethral resection samples. Therefore, pathologists have less experience (and comfort) diagnosing such lesions. Awareness of the diagnostic possibilities for this spectrum of mesenchymal lesions encountered in the prostate provides a significant step toward arriving at the correct diagnosis.

\section{Disclosure/conflict of interest}

The author declare no conflict of interest. 


\section{References}

1 Cheville J, Algaba F, Epstein JI, et al. Mesenchymal tumours. In: Moch H, Humphrey PA, Ulbright TM, et al. (eds). WHO Classification of Tumours of the Urinary System and Male Genital Organs. IARC Press: Lyon, France, 2016;pp 175-177.

2 Bostwick DG, Hossain D, Qian J, et al. Phyllodes tumor of the prostate: long-term followup study of 23 cases. J Urol 2004;172:894-899.

3 Herawi M, Epstein JI. Specialized stromal tumors of the prostate: a clinicopathologic study of 50 cases. Am J Surg Pathol 2006;30:694-704.

4 Gaudin PB, Rosai J, Epstein JI. Sarcomas and related proliferative lesions of specialized prostatic stroma: a clinicopathologic study of 22 cases. Am J Surg Pathol 1998;22:148-162.

5 Sadimin ET, Epstein JI. Round cell pattern of prostatic stromal tumor of uncertain malignant potential: a subtle newly recognized variant. Hum Pathol 2016;52: 68-73.

6 Nagar M, Epstein JI. Epithelial proliferations in prostatic stromal tumors of uncertain malignant potential (STUMP). Am J Surg Pathol 2011;35:898-903.

7 Pan CC, Epstein JI. Common chromosomal aberrations detected by array comparative genomic hybridization in specialized stromal tumors of the prostate. Mod Pathol 2013;26:1536-1543.

8 Fletcher CDM, Bridge JA, Lee J-C. Extrapleural solitary fibrous tumour. In: Fletcher CDM, Bridge JA, Hogendoorn PCW, et al. (eds). WHO Classification of Tumors of Soft Tissue and Bone. IARC Press: Lyon, France, 2013;pp 80-82.

9 Robinson DR, Wu YM, Kalyana-Sundaram S, et al. Identification of recurrent NAB2-STAT6 gene fusions in solitary fibrous tumor by integrative sequencing. Nat Genet 2013;45:180-185.

10 Gharaee-Kermani M, Mehra R, Robinson DR, et al. Complex cellular composition of solitary fibrous tumor of the prostate. Am J Pathol 2014;184:732-739.

11 Cheah AL, Billings SD, Goldblum JR, et al. STAT6 rabbit monoclonal antibody is a robust diagnostic tool for the distinction of solitary fibrous tumour from its mimics. Pathology 2014;46:389-395.

12 Doyle LA, Vivero M, Fletcher CD, et al. Nuclear expression of STAT6 distinguishes solitary fibrous tumor from histologic mimics. Mod Pathol 2014;27: 390-395.

13 Yoshida A, Tsuta K, Ohno M, et al. STAT6 immunohistochemistry is helpful in the diagnosis of solitary fibrous tumors. Am J Surg Pathol 2014;38:552-559.

14 Guner G, Bishop JA, Bezerra SM, et al. The utility of STAT6 and ALDH1 expression in the differential diagnosis of solitary fibrous tumor versus prostate-specific stromal neoplasms. Hum Pathol 2016;54:184-188.

15 Creytens D, Libbrecht L, Ferdinande L. Nuclear expression of STAT6 in dedifferentiated liposarcomas with a solitary fibrous tumor-like morphology: a diagnostic pitfall. Appl Immunohistochem Mol Morphol 2015;23:462-463.

16 Doyle LA, Tao D, Marino-Enriquez A. STAT6 is amplified in a subset of dedifferentiated liposarcoma. Mod Pathol 2014;27:1231-1237.

17 Ullman D, Gordetsky J, Siegal GP, et al. PAX8 expression in solitary fibrous tumor: a potential diagnostic pitfall. Appl Immunohistochem Mol Morphol 2017 [Epub ahead of print July 26].
18 Ronchi A, La Mantia E, Gigantino V, et al. A rare case of malignant solitary fibrous tumor in prostate with review of the literature. Diagn Pathol 2017;12:50.

19 Salas S, Resseguier N, Blay JY, et al. Prediction of local and metastatic recurrence in solitary fibrous tumor: construction of a risk calculator in a multicenter cohort from the French Sarcoma Group (FSG) database. Ann Oncol 2017;28:1979-1987.

20 Demicco EG, Wagner MJ, Maki RG, et al. Risk assessment in solitary fibrous tumors: validation and refinement of a risk stratification model. Mod Pathol 2017;1433-1442.

21 Demicco EG, Park MS, Araujo DM, et al. Solitary fibrous tumor: a clinicopathological study of 110 cases and proposed risk assessment model. Mod Pathol 2012;25:1298-1306.

22 Hossain D, Meiers I, Qian J, et al. Prostatic leiomyoma with atypia: follow-up study of 10 cases. Ann Diagn Pathol 2008;12:328-332.

23 Lee TK, Miyamoto H, Osunkoya AO, et al. Smooth muscle neoplasms of the urinary bladder: a clinicopathologic study of 51 cases. Am J Surg Pathol 2010;34: 502-509.

24 Montgomery EA, Shuster DD, Burkart AL, et al. Inflammatory myofibroblastic tumors of the urinary tract: a clinicopathologic study of 46 cases, including a malignant example inflammatory fibrosarcoma and a subset associated with high-grade urothelial carcinoma. Am J Surg Pathol 2006;30:1502-1512.

25 Ro JY, el-Naggar AK, Amin MB, et al. Pseudosarcomatous fibromyxoid tumor of the urinary bladder and prostate: immunohistochemical, ultrastructural, and DNA flow cytometric analyses of nine cases. Hum Pathol 1993;24:1203-1210.

26 Huang WL, Ro JY, Grignon DJ, et al. Postoperative spindle cell nodule of the prostate and bladder. J Urol 1990;143:824-826.

27 Proppe KH, Scully RE, Rosai J. Postoperative spindle cell nodules of genitourinary tract resembling sarcomas. A report of eight cases. Am J Surg Pathol 1984;8: 101-108.

28 Tsuzuki T, Magi-Galluzzi C, Epstein JI. ALK-1 expression in inflammatory myofibroblastic tumor of the urinary bladder. Am J Surg Pathol 2004;28:1609-1614.

29 McKenney JK.. An approach to the classification of spindle cell proliferations in the urinary bladder. Adv Anat Pathol 2005;12:312-323.

30 Herawi M, Montgomery EA, Epstein JI. Gastrointestinal stromal tumors (GISTs) on prostate needle biopsy: a clinicopathologic study of 8 cases. Am J Surg Pathol 2006;30:1389-1395.

31 Miettinen MM, Corless CL, Debiec-Rychter M, et al. WHO Classification of Tumours of Soft Tissue and Bone In:Fletcher CD, Bridge JA, Hogendoorn PCW, et al. (eds). IARC Press: Lyon, France, 2013; pp 164-166.

32 Hemminger J, Iwenofu OH. Discovered on gastrointestinal stromal tumours 1 (DOG1) expression in nongastrointestinal stromal tumour (GIST) neoplasms. Histopathology 2012;61:170-177.

33 Rane A, Juhasz A, McEwan A, et al. A urological diagnostic conundrum: schwannoma masquerading as an enlarged prostate. Br J Urol 1995;75:683-684.

34 Latchamsetty KC, Elterman L, Coogan CL. Schwannoma of a seminal vesicle. Urology 2002;60:515.

35 He R, Yang X, Li X, et al. Cystic schwannoma of a seminal vesicle. J Androl 2012;33:798-800. 
36 Ud Din N, Ahmad Z, Ahmed A. Schwannomas with pseudoglandular elements: clinicopathologic study of 61 cases. Ann Diagn Pathol 2016;20:24-28.

37 Kanthan GL, Izard MA, Emmett L, et al. Schwannoma Showing Avid Uptake on 68Ga-PSMA-HBED-CC PET/ CT. Clin Nucl Med 2016;41:703-704.

38 Rischpler C, Maurer T, Schwaiger M, et al. Intense PSMA-expression using (68)Ga-PSMA PET/CT in a paravertebral schwannoma mimicking prostate cancer metastasis. Eur J Nucl Med Mol Imaging 2016;43: 193-194

39 Wang W, Tavora F, Sharma R, et al. PSMA expression in Schwannoma: a potential clinical mimicker of metastatic prostate carcinoma. Urol Oncol 2009;27: $525-528$.

40 Perrone T, Carson LF, Dehner LP. Rhabdomyosarcoma with heterologous cartilage of the uterine cervix: a clinicopathologic and immunohistochemical study of an aggressive neoplasm in a young female. Med Pediatr Oncol 1990;18:72-76.

41 Daya DA, Scully RE. Sarcoma botryoides of the uterine cervix in young women: a clinicopathological study of 13 cases. Gynecol Oncol 1988;29:290-304.

42 Harel M, Ferrer FA, Shapiro LH, et al. Future directions in risk stratification and therapy for advanced pediatric genitourinary rhabdomyosarcoma. Urol Oncol 2016;34: 103-115.

43 Children's Oncology Group. Rhabdomyosarcoma (www.childrensoncologygroup.org.).

44 Hansel DE, Epstein JI.. Sarcomatoid carcinoma of the prostate: a study of 42 cases. Am J Surg Pathol 2006;30: 1316-1321.

45 Shannon RL, Ro JY, Grignon DJ, et al. Sarcomatoid carcinoma of the prostate. A clinicopathologic study of 12 patients. Cancer 1992;69:2676-2682.

46 Wick MR, Young RH, Malvesta R, et al. Prostatic carcinosarcomas. Clinical, histologic, and immunohistochemical data on two cases, with a review of the literature. Am J Clin Pathol 1989;92:131-139.

47 Cheng L, Bostwick DG. Atypical sclerosing adenosis of the prostate: a rare mimic of adenocarcinoma. Histopathology 2010;56:627-631.

48 Jones EC, Clement PB, Young RH. Sclerosing adenosis of the prostate gland. A clinicopathological and immunohistochemical study of 11 cases. Am J Surg Pathol 1991;15:1171-1180.

49 Young RH, Clement PB. Sclerosing adenosis of the prostate. Arch Pathol Lab Med 1987;111: $363-366$.

50 Mavrogenis AF, Pala E, Guerra G, et al. Post-radiation sarcomas. Clinical outcome of 52 patients. J Sur Oncol 2012;105:570-576.
51 Laskin WB, Silverman TA, Enzinger FM. Postradiation soft tissue sarcomas. An analysis of 53 cases. Cancer 1988;62:2330-2340.

52 Lopez-Beltran A, Eble JN, Bostwick DG. Pleomorphic giant cell carcinoma of the prostate. Arch Pathol Lab Med 2005;129:683-685.

53 Reikie BA, Yilmaz A, Medlicott S, et al. Mixed epithelial-stromal tumor (MEST) of seminal vesicle: a proposal for unified nomenclature. Adv Anat Pathol 2015;22:113-120.

54 Paner GP, Lopez-Beltran A, So JS, et al. Spectrum of cystic epithelial tumors of the prostate: most cystadenocarcinomas are ductal type with intracystic papillary pattern. Am J Surg Pathol 2016;40:886-895.

55 Ortiz-Rey JA, Juaneda-Benavides L, Peteiro-Cancelo A, et al. Another application of PAX8: to confirm the presence of seminal vesicle epithelium in prostate needle biopsies. Appl Immunohistochem Mol Morphol 2015;23:161-162.

56 Wang G, Black PC, Skinnider BF, et al. Post-radiation epithelioid angiosarcoma of the urinary bladder and prostate. Can Urol Assoc J 2016;10:E197-E200.

57 Ristau BT, Tomaszewski JJ, Parwani AV, et al. Incidentally discovered capillary hemangioma of the prostate. Can J Urol 2011;18:5914-5915.

58 Serizawa RR, Norgaard N, Horn T, et al. Hemangioma of the prostate-an unusual cause of lower urinary tract symptoms: case report. BMC Urol 2011;11:4.

59 Iyer A, Thompson L, Cooper K. Epithelioid haemangioendothelioma, mimicking prostatic adenocarcinoma. Histopathology 2012;60:522-524.

60 Kulmala RV, Seppanen JH, Vaajalahti PJ, et al. Malignant fibrous histiocytoma of the prostate. Case report. Scand J Urol Nephrol 1994;28:429-431.

61 Oesterling JE, Epstein JI, Brendler CB. Myxoid malignant fibrous histiocytoma of the bladder. Cancer 1990;66:1836-1842.

62 Pan CC, Chang YH. Primary synovial sarcoma of the prostate. Histopathology 2006;48:321-323.

63 Hsieh KL, Lu CC, Li CF, et al. malignant peripheral nerve sheath tumor of prostate: a rare case report and literature review. Case Rep Urol 2016;2016:9317567.

64 Rames RA, Smith MT. Malignant peripheral nerve sheath tumor of the prostate: a rare manifestion of neurofibromatosis type 1. J Urol 1999;162:165-166.

65 Jana M, Gamanagatti S, Kumar R, et al. Pelvic neurofibroma arising from prostate in a case of neurofibromatosis-1. Indian J Urol 2011;27:415-417.

66 Perez Valcarcel J, Sanchez Arca ME, Sousa Escandon A, et al. [Granular cell tumour of prostate diagnosed by transrectal biopsy. Which therapeutic attitude?]. Actas urol Esp 2006;30:1031-1033. 